\title{
Parton Distribution Functions from Lattice QCD
}

\author{
Jianhui Zhang* \\ Institut für Theoretische Physik, Universität Regensburg, D-93040 Regensburg, Germany \\ E-mail: jianhui.zhang@ur.de
}

Parton distribution functions (PDFs) are important quantities that characterize the structure of hadrons at high energy. Calculating them from first principles has been a long standing challenge in hadron physics and particle physics. In the past few years, rapid progress has been made in directly computing PDFs from lattice QCD. Here I summarize the current status of lattice calculation of PDFs. The state-of-the-art lattice results on nucleon PDFs have shown a reasonable agreement with phenomenological results extracted from the experimental data.

XXVII International Workshop on Deep-Inelastic Scattering and Related Subjects - DIS2019

8-12 April, 2019

Torino, Italy

${ }^{*}$ Speaker. 


\section{Introduction}

Parton distribution functions (PDFs) are important quantities describing the momentum density of quarks and gluons inside a hadron. In the field-theoretic language, they are defined as the expectation value of lightcone correlations in the hadron state. How to calculate them from first principles has been a long-standing problem in hadron physics. In the past few decades, the ab initio lattice QCD approach has only been able to access the first few moments of the PDFs [1, 2], while reconstructing the full distribution requires information on all their moments. Phenomenologically, the PDFs are usually determined by assuming a smoothly parametrized form and fitting the unknown parameters to a large variety of experimental data (for a recent review, see e.g. Ref. [3]).

Recently, a new theory framework, which is now known as large momentum effective theory (LaMET) $[4,5]$, has been developed that allows for a lattice calculation of the full $x$-dependence, instead of the moments, of the PDFs. Since LaMET was proposed, much progress has been achieved both in the theoretical understanding of the formalism and in the direct calculation of the PDFs from lattice QCD (see a recent review [6] and references therein). Despite limited volumes and relatively coarse lattice spacings, the state-of-the-art nucleon isovector quark PDFs determined from lattice data at the physical point have shown a reasonable agreement $[7,8,9]$ with phenomenological results extracted from the experimental data $[10,11,12,13,14]$. Of course, a careful study of theoretical uncertainties and lattice artifacts is still needed to fully establish the reliability of the results.

In this talk, I review recent progress in lattice calculations of the PDFs, with a particular focus on the isovector quark PDFs in the proton, for which intensive studies have been carried out in the past few years.

\section{From quasi-PDF to PDF}

The leading-twist quark PDF in the proton is defined as

$$
q(x, \mu)=\int \frac{d \xi^{-}}{4 \pi} e^{-i x P^{+} \xi^{-}}\left\langle P\left|\bar{\psi}\left(\xi^{-}\right) \Gamma W\left(\xi^{-}, 0\right) \psi(0)\right| P\right\rangle,
$$

where the proton has momentum $P^{\mu}=\left(P^{0}, 0,0, P^{z}\right), \psi, \bar{\psi}$ denote the quark fields, $\xi^{ \pm}=(t \pm z) / \sqrt{2}$ are the lightcone coordinates, $x$ is the fraction of proton momentum carried by the quark, $\Gamma=$ $\left\{\gamma^{+}, \gamma^{+} \gamma_{5}, \gamma^{+} \gamma^{\perp} \gamma_{5}\right\}$ is a Dirac structure that specifies the leading-twist quark PDF, $\mu$ is the renormalization scale in the $\overline{\mathrm{MS}}$ scheme, and

$$
W\left(\xi^{-}, 0\right)=\exp \left(-i g \int_{0}^{\xi^{-}} d \eta^{-} A^{+}\left(\eta^{-}\right)\right)
$$

is the Wilson line inserted to ensure gauge invariance of the nonlocal quark correlator, where $A^{+}=$ $A_{a}^{+} t^{a}$ with $t^{a}$ being the generators in the fundamental representation of color $S U$ (3) group.

According to LaMET, a well-suited quasi-PDF candidate that can be used to extract the above quark PDF is given by

$$
\tilde{q}\left(x, \tilde{\mu}, P^{z}\right)=N \int \frac{d z}{4 \pi} e^{i z x P^{z}}\langle P|\bar{\psi}(z) \Gamma W(z, 0) \psi(0)| P\rangle,
$$


where $z$ is a spatial direction, $\Gamma=\left\{\gamma^{t}, \gamma^{z} \gamma_{5}, \gamma^{t} \gamma^{\perp} \gamma_{5}\right\}$ is a Dirac matrix with the corresponding normalization factor $N=P^{z} / P^{t}, \tilde{\mu}$ is the renormalization scale in an appropriate scheme. As shown in Refs. [15, 16, 17], the renormalization of the above quark quasi-PDFs has a multiplicative form in coordinate space so that the matrix elements at different $z$ do not mix with each other. Moreover, the above choice has the advantage of avoiding $\mathscr{O}(1)$ mixing with other PDFs when a non-chiral lattice fermion is used $[18,19]$.

The renormalization of the above quark quasi-PDF can be carried out nonperturbatively in the RI/MOM scheme [20, 21]. After the bare matrix element on the r.h.s. of Eq. 2.3, denoted as $\tilde{h}\left(z, P^{z}, a\right)$, is calculated on the lattice, it can be renormalized by demanding that the counterterm cancels all the loop contributions for the matrix element of the same operator in an off-shell external quark state at a specific momentum [20,21]:

$$
\tilde{h}_{R}\left(z, P^{z}, \mu_{R}, p_{z}^{R}\right)=Z^{-1}\left(z, \mu_{R}, p_{z}^{R}, a\right) \tilde{h}\left(z, P^{z}, a\right), \quad Z\left(z, \mu_{R}, p_{z}^{R}, a\right)=\left.\frac{\operatorname{Tr}\left[\Lambda_{\Gamma} \mathscr{P}\right]}{\operatorname{Tr}\left[\Lambda_{\Gamma} \mathscr{P}\right]_{\text {tree }}}\right|_{\substack{p^{2}=-\mu_{R}^{2} \\ p_{z}=p_{z}^{2}}},
$$

where $\Lambda_{\Gamma}$ is the amputated Green's function of the quark bilinear operator in Eq. (2.3) in an offshell quark state with momentum $p . \mu_{R}, p_{z}^{R}$ are renormalization scales introduced in the RI/MOM scheme. $\mathscr{P}$ is a projection operator that defines the renormalization factor [21, 8, 22]. After renormalization, $\tilde{h}_{R}$ has a well-defined continuum limit. It can be converted to the renormalized quasi-PDF, $\tilde{q}_{R}$, by the Fourier transform in Eq. (2.3). When taking the isovector $u-d$ combination, $\tilde{q}_{R}$ can be connected to the normal PDF in the $\overline{\mathrm{MS}}$ scheme through the following factorization formula

$$
\tilde{q}_{R}\left(x, P_{z}, \mu_{R}, p_{z}^{R}\right)=\int_{-1}^{1} \frac{d y}{|y|} C\left(\frac{x}{y}, \frac{\mu_{R}}{p_{z}^{R}}, \frac{y P_{z}}{\mu}, \frac{y P_{z}}{p_{z}^{R}}\right) q(y, \mu)+\mathscr{O}\left(\frac{M^{2}}{P_{z}^{2}}, \frac{\Lambda_{\mathrm{QCD}}^{2}}{x^{2}(1-x) P_{z}^{2}}\right),
$$

with a perturbatively calculable hard matching kernel $C$, where the higher-twist contributions behave like $1 /\left[x^{2}(1-x) P_{z}^{2}\right]$ instead of $1 / P_{z}^{2}$, as demonstrated in Ref. [23].

\section{Lattice results on PDFs}

Within the LaMET approach, the isovector quark unpolarized, helicity and transversity PDFs have been computed by two different groups: $\operatorname{LP}^{3}[7,8,22]$ and ETMC $[9,24]$. In the work of $\mathrm{LP}^{3}$, the calculation was done using clover valence fermions on an ensemble of gauge configurations with lattice spacing $a=0.09 \mathrm{fm}$, box size $L \approx 5.8 \mathrm{fm}$ and pion mass $M_{\pi} \approx 135 \mathrm{MeV}$ with $N_{f}=2+1+1$ (degenerate up/down, strange and charm) flavors of highly improved staggered quarks (HISQ) [25] generated by MILC Collaboration [26]. The gauge links were hypercubic (HYP)-smeared [27] to improve the discretization effects. The calculation of ETMC used gauge configurations generated with the Iwasaki improved gluon action $[28,29]$ and the twisted mass fermion action with clover improvement [30,31]. Their results were obtained with an ensemble of two degenerate light quarks $\left(N_{f}=2\right)$ at maximal twist, with quark masses that were tuned to reproduce approximately the physical pion mass value [32]. The lattice spacing was $a=0.094 \mathrm{fm}$, box size $L \approx 4.5 \mathrm{fm}$ and pion mass $M_{\pi} \approx 130 \mathrm{MeV}$. They applied stout smearing [33] to the links of the Wilson line entering the operator, and tested up to 20 smearing steps and found complete agreement upon renormalization. Both groups have used a momentum smearing [34] that was designed 

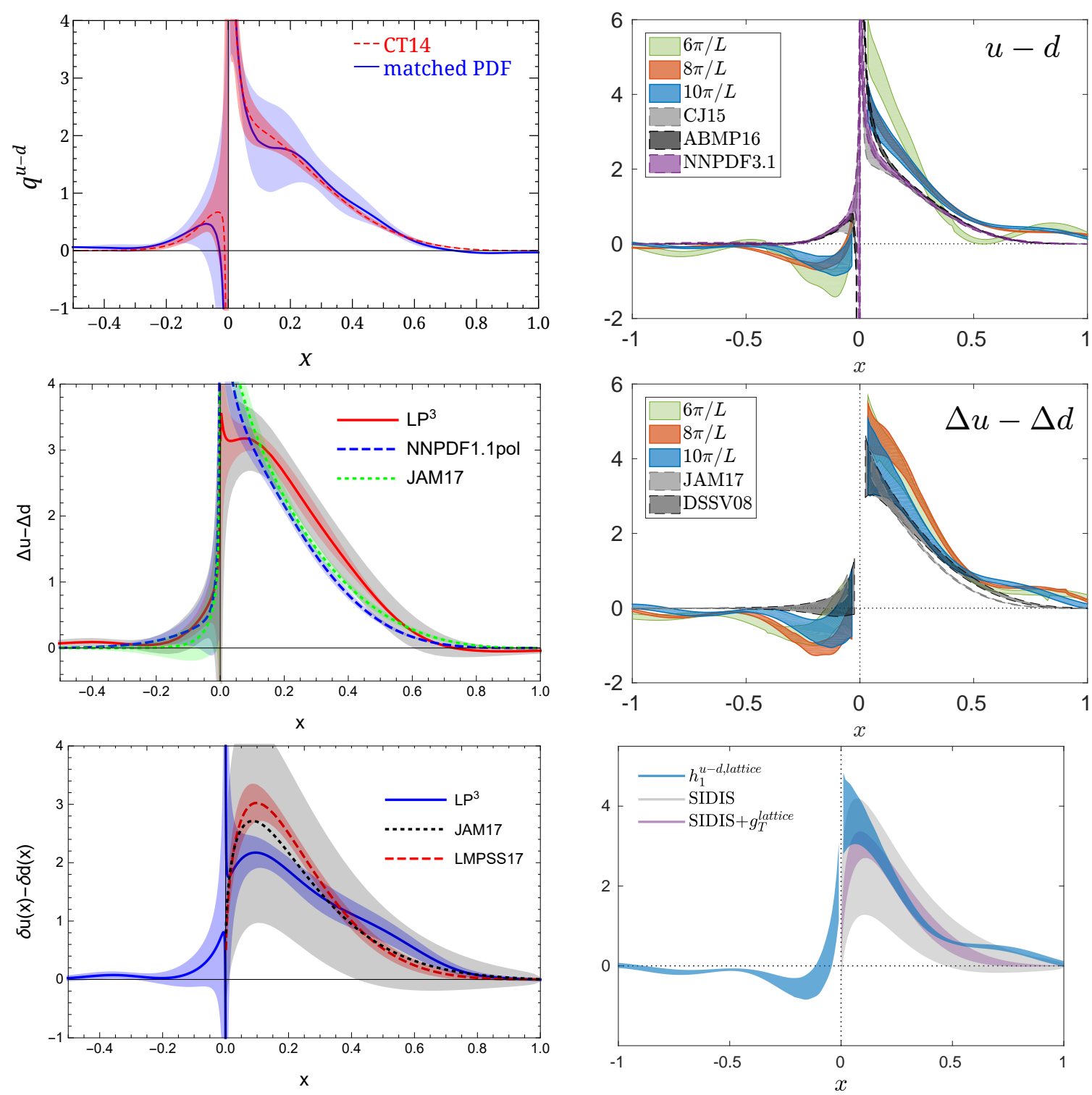

Figure 1: Comparison of isovector unpolarized (upper row), helicity (middle row) and transversity (lower row) quark distributions with phenomenological results (left: $\mathrm{LP}^{3}$, right: ETMC).

to align the overlap with nucleons of the desired boost momentum, and the largest momentum reached was 3.0 and $1.4 \mathrm{GeV}$, respectively.

As can be seen from the ETMC plot in Fig. 1, the lattice results tend to approach the phenomenological curves when the proton momentum is increased. In addition, there is an oscillatory behavior in the ETMC plot, which is due to the truncated Fourier transform from coordinate space matrix elements to momentum space distributions. In the $\mathrm{LP}^{3}$ plot such an oscillation is hardly visible because a "derivative method" [35] has been applied to suppress the unphysical oscillation effects.

In all three cases, the $\mathrm{LP}^{3}$ results show a reasonable agreement with phenomenological curves 
within errors. This is encouraging. However, one should be aware that in these plots a thorough analysis of the systematic uncertainties is still missing, which needs to be done in the future to fully establish the reliability of these results.

\section{References}

[1] W. Detmold, W. Melnitchouk and A. W. Thomas, Parton distributions from lattice QCD, Eur. Phys. J.direct 3 (2001) 13, [hep-lat/ 0108002 ].

[2] LHPC, TXL collaboration, D. Dolgov et al., Moments of nucleon light cone quark distributions calculated in full lattice QCD, Phys. Rev. D66 (2002) 034506, [hep-lat/ 0201021 ].

[3] J. Gao, L. Harland-Lang and J. Rojo, The Structure of the Proton in the LHC Precision Era, Phys. Rept. 742 (2018) 1-121, [1709.04922].

[4] X. Ji, Parton Physics on a Euclidean Lattice, Phys. Rev. Lett. 110 (2013) 262002, [1305 . 1539].

[5] X. Ji, Parton Physics from Large-Momentum Effective Field Theory, Sci. China Phys. Mech. Astron. 57 (2014) 1407-1412, [1 404 . 6680].

[6] K. Cichy and M. Constantinou, A guide to light-cone PDFs from Lattice QCD: an overview of approaches, techniques and results, 1811.07248.

[7] J.-W. Chen, L. Jin, H.-W. Lin, Y.-S. Liu, Y.-B. Yang, J.-H. Zhang et al., Lattice Calculation of Parton Distribution Function from LaMET at Physical Pion Mass with Large Nucleon Momentum, 1803.04393.

[8] H.-W. Lin, J.-W. Chen, L. Jin, Y.-S. Liu, Y.-B. Yang, J.-H. Zhang et al., Spin-Dependent Parton Distribution Function with Large Momentum at Physical Pion Mass, 1807.07431.

[9] C. Alexandrou, K. Cichy, M. Constantinou, K. Jansen, A. Scapellato and F. Steffens, Reconstruction of light-cone parton distribution functions from lattice QCD simulations at the physical point, 1803.02685.

[10] S. Dulat, T.-J. Hou, J. Gao, M. Guzzi, J. Huston, P. Nadolsky et al., New parton distribution functions from a global analysis of quantum chromodynamics, Phys. Rev. D93 (2016) 033006, [1506. 07443].

[11] NNPDF collaboration, R. D. Ball et al., Parton distributions from high-precision collider data, Eur. Phys. J. C77 (2017) 663, [1706.00428].

[12] L. A. Harland-Lang, A. D. Martin, P. Motylinski and R. S. Thorne, Parton distributions in the LHC era: MMHT 2014 PDFs, Eur. Phys. J. C75 (2015) 204, [1412. 3989].

[13] NNPDF collaboration, E. R. Nocera, R. D. Ball, S. Forte, G. Ridolfi and J. Rojo, A first unbiased global determination of polarized PDFs and their uncertainties, Nucl. Phys. B887 (2014) 276-308, [1406.5539].

[14] J. J. Ethier, N. Sato and W. Melnitchouk, First simultaneous extraction of spin-dependent parton distributions and fragmentation functions from a global QCD analysis, Phys. Rev. Lett. 119 (2017) 132001, [1705.05889].

[15] X. Ji, J.-H. Zhang and Y. Zhao, Renormalization in Large Momentum Effective Theory of Parton Physics, Phys. Rev. Lett. 120 (2018) 112001, [1706.08962].

[16] T. Ishikawa, Y.-Q. Ma, J.-W. Qiu and S. Yoshida, Renormalizability of quasiparton distribution functions, Phys. Rev. D96 (2017) 094019, [1707.03107]. 
[17] J. Green, K. Jansen and F. Steffens, Nonperturbative renormalization of nonlocal quark bilinears for quasi-PDFs on the lattice using an auxiliary field, Phys. Rev. Lett. 121 (2018) 022004, [1707.07152].

[18] M. Constantinou and H. Panagopoulos, Perturbative renormalization of quasi-parton distribution functions, Phys. Rev. D96 (2017) 054506, [1705.11193].

[19] J.-W. Chen, T. Ishikawa, L. Jin, H.-W. Lin, Y.-B. Yang, J.-H. Zhang et al., Operator classification for nonlocal quark bilinear on lattice, 1710.01089.

[20] J.-W. Chen, T. Ishikawa, L. Jin, H.-W. Lin, Y.-B. Yang, J.-H. Zhang et al., Parton distribution function with nonperturbative renormalization from lattice QCD, Phys. Rev. D97 (2018) 014505, [1706.01295].

[21] I. W. Stewart and Y. Zhao, Matching the quasiparton distribution in a momentum subtraction scheme, Phys. Rev. D97 (2018) 054512, [1709.04933].

[22] Y.-S. Liu, J.-W. Chen, L. Jin, R. Li, H.-W. Lin, Y.-B. Yang et al., Nucleon Transversity Distribution at the Physical Pion Mass from Lattice QCD, 1810.05043.

[23] V. M. Braun, A. Vladimirov and J.-H. Zhang, Power corrections and renormalons in parton quasi-distributions, 1810.00048.

[24] C. Alexandrou, K. Cichy, M. Constantinou, K. Jansen, A. Scapellato and F. Steffens, Transversity parton distribution functions from lattice QCD, 1807.00232.

[25] HPQCD, UKQCD collaboration, E. Follana, Q. Mason, C. Davies, K. Hornbostel, G. P. Lepage, J. Shigemitsu et al., Highly improved staggered quarks on the lattice, with applications to charm physics, Phys. Rev. D75 (2007) 054502, [hep-lat/ 0610092 ].

[26] MILC collaboration, A. Bazavov et al., Lattice QCD ensembles with four flavors of highly improved staggered quarks, Phys. Rev. D87 (2013) 054505, [1212 .4768].

[27] A. Hasenfratz and F. Knechtli, Flavor symmetry and the static potential with hypercubic blocking, Phys. Rev. D64 (2001) 034504, [hep-lat/ 010302 ]].

[28] Y. Iwasaki, Renormalization Group Analysis of Lattice Theories and Improved Lattice Action. II. Four-dimensional non-Abelian SU(N) gauge model, 1111.7054.

[29] A. Abdel-Rehim et al., A first look at maximally twisted mass lattice QCD calculations at the physical point, PoS LATTICE2013 (2014) 264, [1311 . 4522].

[30] R. Frezzotti and G. C. Rossi, Chirally improving Wilson fermions. 1. O(a) improvement, JHEP 08 (2004) 007, [hep-lat/0306014].

[31] B. Sheikholeslami and R. Wohlert, Improved Continuum Limit Lattice Action for QCD with Wilson Fermions, Nucl. Phys. B259 (1985) 572.

[32] ETM collaboration, A. Abdel-Rehim et al., First physics results at the physical pion mass from $N_{f}=2$ Wilson twisted mass fermions at maximal twist, Phys. Rev. D95 (2017) 094515, [1507. 05068 ].

[33] C. Morningstar and M. J. Peardon, Analytic smearing of SU(3) link variables in lattice QCD, Phys. Rev. D69 (2004) 054501, [hep-lat/0311018].

[34] G. S. Bali, B. Lang, B. U. Musch and A. Schäfer, Novel quark smearing for hadrons with high momenta in lattice QCD, Phys. Rev. D93 (2016) 094515, [1602 . 05525].

[35] H.-W. Lin, J.-W. Chen, T. Ishikawa and J.-H. Zhang, Improved Parton Distribution Functions at Physical Pion Mass, 1708.05301. 